\title{
Alternativas y acciones en el tema de residuos sólidos planteadas por las municipalidades de Jiménez y Oreamuno y su relación con el desarrollo y la sostenibilidad (Artículo informativo)
}

Alternatives and actions regarding solid residues, presented by Jimenez and Oreamuno municipalities and their relation to development and sustainability (Informative)

Rooel Campos-Rodríguez'

Noemy Quiros-Bustos²

Alfonso Navarro-Garro ${ }^{3}$

Fecha de recepción: 04 de agosto del 2012

Fecha de aprobación: 17 de diciembre del 2012

Campos-Rodríguez, R.; Quirós-Bustos, N.; NavarroGarro, A. Alternativas y acciones en el tema de residuos sólidos planteadas por las municipalidades de Jiménez y Oreamuno y su relación con el desarrollo y la sostenibilidad. Tecnología en Marcha. Vol. 26, Nº 2. Pág |04-I I |

Profesor-Investigador. Escuela de Ingeniería Agropecuaria Administrativa, ITCR. rocampos@itcr.ac.cr

2 Profesora-Investigadora. Escuela de Química, ITCR. nquiros@itcr. ac.cr.

3 Profesor-Investigador. Escuela de Ingeniería en Seguridad Laboral e Higiene Ambiental, ITCR. alnavarro@itcr.ac.cr 


\section{Palabras clave}

Residuos sólidos; empoderamiento; sensibilidad; desarrollo sostenible

\section{Resumen}

Los procesos productivos no son totalmente eficientes, lo cual contribuye a la generación de residuos. En Costa Rica, son las municipalidades las responsables del manejo integral de los residuos sólidos. Es por eso que la problemática de esta investigación se centra en el manejo de tales desechos, que se ha convertido en uno de los principales problemas ambientales que enfrentan los municipios del país. Es necesario hacerle frente desde la perspectiva del desarrollo sostenible, para lo cual se requiere una nueva visión y sensibilización de los gobiernos locales y de los habitantes ante esta problemática.

Un ejemplo de cómo encarar la problemática ambiental es la labor que están desarrollando los municipios de Jiménez y Oreamuno. Estas dos instituciones han trabajado en aspectos de educación ambiental, desarrollo y sostenibilidad y han logrado resultados favorables en el tratamiento y disposición adecuada de los residuos sólidos.

El municipio de Oreamuno cuenta con un plan de manejo integral de residuos sólidos, tanto orgánicos como inorgánicos. También tiene un centro de acopio y se está trabajando en la implementación de biorreactores que permitirán la producción de bioabonos, captación de gas metano, remoción de metales en los lixiviados, así como la recuperación, comercialización y transformación de los residuos reciclables y reutilizables. Por su parte, el municipio de Jiménez realiza un manejo adecuado de los residuos; los desechos inorgánicos reciclables son aprovechados por medio del centro de acopio del cantón y los residuos orgánicos son transformados en abono orgánico, el cual se utiliza en las fincas de la localidad. El objetivo de este artículo es analizar estos planteamientos, así como su relación con el desarrollo y la sostenibilidad.

\section{Key words}

Solid waste; empowerment; sensitivity; sustainable development

\begin{abstract}
Currently, the production processes are not efficient, which contributes to the generation of waste. In Costa Rica the municipalities are responsible for the integrated management of solid waste. That is why this research focuses on issues of waste management which has become a major environmental problem. So, it is necessary to confront these issues from the perspective of sustainable development, requiring a new vision and awareness of people regarding the environment and the problem of solid waste.
\end{abstract}

An example of dealing with environmental problems is the work that is being developed in the towns of Jiménez and Oreamuno. These municipalities have worked on environmental education, development and sustainability, and the results have been favorable for treatment and proper disposal of solid waste.

In Oreamuno, there is a management plan for organic and inorganic solid waste. There is a storage center and the town is expected to implement in the future bioreactors that enable the production of biofertilizers, methane gas collection, removal of metals in the leachate, and the recovery, processing and marketing of recyclable and reusable waste. Meanwhile, the town of Jiménez has a waste management system, recyclable inorganic wastes are utilized in the collection center and organic waste is transformed into organic fertilizer, which is used on farms in the locality. Therefore, the aim of this paper is to analyze these approaches and their relationship to development and sustainability. 


\section{Introducción}

Los seres humanos, con el objetivo de satisfacer sus gustos y preferencias, se han visto en la necesidad de aumentar los niveles de producción para compensar el incremento en el consumo. Esto ha provocado que se establezcan sistemas complejos de economía, producción y tecnología, en los cuales, a pesar de los avances existentes en este último campo, los procesos productivos todavía no son totalmente eficientes, contribuyendo a la generación de residuos. El crecimiento económico debe estar presente en la sociedad y para esto se debe impulsar un modelo económico que permita la generación de procesos productivos, que pongan en manos de los consumidores una gama variada de bienes y servicios para satisfacer sus necesidades, pero está claro que los residuos sólidos serán parte de este proceso expansivo de crecimiento.

En Costa Rica, las municipalidades son las responsables del manejo integral de los residuos sólidos, según la Ley para la Gestión Integral de Residuos (Soto, 20l0). Sin embargo, durante las últimas tres décadas, el crecimiento en la generación y la variedad de tipos de residuos sólidos ha aumentado de manera significativa (Programa Estado de la Nación, 2006), provocando que algunos municipios no logren cumplir con este compromiso.

Soto (2010) afirma que en el caso de Costa Rica, estudios conducidos por municipios rurales en el año 2009, como el de Orotina, establecen un índice de generación de 1 kg/persona/día; mientras que para el cantón central de San José el dato es de I,024 kg/persona/día.

En las comunidades rurales la problemática es mayor, debido a los bajos presupuestos con que cuentan los municipios, la falta de sitios adecuados para la disposición final, centros de acopio para la valorización de los residuos, el no pago por el servicio de recolección de residuos, así como modelos de acompañamiento que permitan a las comunidades lograr un acercamiento científico ante el tema de los residuos sólidos que derive en soluciones integrales, que surjan de los propios pobladores del municipio y que sean sostenibles en el tiempo (Soto 2006, 2010).

Novo (2009) propone que una forma de hacerle frente a las problemáticas ambientales es entendiendo la sostenibilidad como un fin de corto, mediano y largo plazo, que permite allanar el camino para que futuras generaciones disfruten de una buena calidad de vida y mediante un nuevo paradigma que tome en cuenta criterios ambientales, sociales, económicos y culturales, y así se pueda contrarrestar el cambio global que se está viviendo por medio de un desarrollo sostenible. Para Caduto (1992), lo anterior se puede lograr en la medida en que se tengan conocimientos, aptitudes, actitudes, deseos y motivación, aspectos preponderantes para trabajar individual y colectivamente en la exploración de nuevas soluciones para los problemas actuales, situación que se puede atenuar en el tanto se incluyan programas de educación ambiental en las propuestas de desarrollo.

Todo lo anterior será posible de alcanzar con una participación activa de las comunidades, por lo que se hace necesaria la promoción de instrumentos metodológicos que fortifiquen y promuevan la capacidad con que cuentan las organizaciones para que tengan un control de los procesos, a partir de su propia idiosincrasia e interés de transitar por un cambio social, ambiental y cultural (López, López y Rodríguez, 2005).

Para lograrlo, es necesario conocer la visión de los habitantes sobre el entorno y la problemática de los residuos sólidos, lo cual significa determinar la sensibilización de la comunidad (García, 2006). En tal sentido, los cantones de Jiménez y Oreamuno, ubicados en la provincia de Cartago, han trabajado en elementos como los conocimientos, habilidades, destrezas, actitudes, sensibilidad y empoderamiento para que, por medio de un modelo de Acción Ambiental Positiva, se logren resultados favorables respecto al tratamiento y disposición adecuada de los residuos sólidos.

En este artículo se analizan estos planteamientos, así como su relación con el desarrollo y la sostenibilidad.

\section{Metodología}

Los temas tratados se abordaron siguiendo el tipo de investigación mixta, durante la cual se recopiló información documental y se complementó con datos obtenidos en trabajo de campo.

Las fuentes documentales se obtuvieron de bases de datos pertenecientes a universidades públicas. Entre estos cabe destacar el Repositorio del Instituto Tecnológico de Costa Rica (ITCR), EBSCOhost 
Web, ProQuest y SpringerLink. También se consultaron las bases de datos de Dialnet y Scielo, que incluyen revistas electrónicas.

Los datos de campo se obtuvieron por medio de visitas a las dos municipalidades en estudio, recopilando información a través de entrevistas a funcionarios, quienes manifestaron que por medio de talleres participativos lograron que las comunidades se sensibilizaran ante el tema, despertaran a su realidad y, una vez claros ante la problemática, sacaran a flote sus habilidades y destrezas. Se buscó que las personas, como producto de sus conocimientos, lograran un cambio de actitud y propusieran alternativas, lo que se refleja en el empoderamiento logrado, por lo que se está en presencia de un Modelo de Acción Ambientalmente Positiva.

Para sustentar las afirmaciones que se obtuvieron de las entrevistas y los talleres se hizo una triangulación de la información, comparando los datos obtenidos con la teoría.

Para este caso, la variable gestión de residuos sólidos es la dependiente, mientras que los conocimientos, habilidades y actitudes para la gestión de los residuos sólidos se convierten en las variables independientes, con el fin de demostrar que las comunidades concientizadas y empoderadas de sus recursos son capaces de realizar, en conjunto con los actores institucionales, la gestión integral de los residuos sólidos generados.

\section{Resultados y discusión}

La generación de residuos sólidos ha ido creciendo exponencialmente, no solo en Costa Rica sino en el resto del mundo en las últimas décadas. Algunos de los factores que han contribuido a una mayor generación de residuos en los últimos años son la cultura de usar y tirar, los llamados "desechables", la alta cantidad de los diferentes materiales de empaque, además de una cada vez más arraigada cultura de consumismo y un mayor volumen de importación de productos extranjeros (CYMA, 2007).

Es mediante la Gestión Integral de Residuos Sólidos Municipales que los gobiernos locales pueden propiciar mecanismos de producción limpios, integrando herramientas tecnológicas, administrativas y modernas, logrando la participación responsable de cada uno de los diversos sectores de la sociedad y el fortalecimiento de las instituciones, en un proceso liderado por cada municipalidad que responda a una gestión ambiental solidaria (Gaviria y Soto, 2008).

Un ejemplo de este tipo de iniciativas es la labor que se está desarrollando en los municipios de Jiménez y Oreamuno, donde, reconociendo que pueden existir barreras actitudinales, conceptuales y de control que según Mata et al. (2009) son obstáculos que podrían hacer que las personas no actúen en pro del ambiente, han trabajado en ellas con el propósito de obtener una gestión integral de los residuos sólidos. Es por esto que el cantón de Oreamuno cuenta con un Plan de Manejo Integral de Residuos Sólidos que tiene como objetivo principal "reducir al mínimo los desechos que deban de ser depositados en el relleno sanitario, recuperar los gases y remover los metales en los lixiviados mediante el uso de métodos químicos y biológicos, además de la recuperación, comercialización y transformación de los desechos reciclables y reutilizables, generando oportunidades de empleo a los ciudadanos" (Redondo, 2009).

En vista de lo anterior, el pilar ambiental debe privar ante las leyes económicas y sociales, por tal motivo se hace necesario el cambio en la cultura y el comportamiento ambiental, a través de aspectos como valores ambientales, conocimientos y creencias sobre el ambiente, los cuales a su vez se pueden transformar en habilidades, procedimientos, actitudes y sensibilidad en busca de un empoderamiento de parte de un individuo o grupo de personas para que actúen a favor del ambiente (Mata, Zúñiga, Brenes, Carrillo, Charpentier, Hernández y Zúñiga, 2002).

Lo propuesto anteriormente fue logrado en gran medida por el municipio de Oreamuno mediante talleres participativos, donde los actores involucrados recibieron datos que transformaron en conocimientos, se sensibilizaron ante la problemática de los residuos sólidos y en forma conjunta con las autoridades municipales construyeron una matriz de alternativas, tomando en cuenta aspectos técnicos, ambientales, sociales, culturales, económicos y políticos, lo que derivó en un incremento del acervo cognitivo de todos los participantes, fortaleciendo un pensamiento crítico que, conjuntamente con un cambio de actitud, desencadenó una serie de acontecimientos que se transformaron en propuestas viables para la comunidad. 
Para poder alcanzar la sostenibilidad del sistema, se pretende que la población realice una selección en la fuente, clasificando los residuos sólidos en orgánicos e inorgánicos. En el caso de los residuos orgánicos, mediante la colaboración de las cuatro universidades estatales de Costa Rica, se está trabajando en un plan piloto de diseño y funcionamiento de biorreactores con el cual se podrá contribuir en la elaboración de bioabono, recolección de gas metano y tratamiento de lixiviados. Este aprovechamiento de materiales contribuirá al sistema socioecológico, ya que los materiales se utilizarán en el mejoramiento del recurso suelo para las cosechas dada la vocación agrícola de la zona, impactando de manera positiva el medio ambiente y la salud de los pobladores por la disminución del uso de fertilizantes.

En cuanto a los residuos inorgánicos, el municipio cuenta con un Centro de Acopio, inaugurado a mediados de 2010, que se espera brinde oportunidades laborales a los ciudadanos del cantón que se encuentran dentro de algún grupo de interés social, como personas con algún grado de discapacidad o mujeres jefas de hogar, lo que sin duda alguna es un aporte muy significativo al componente social y económico desde la perspectiva de la sostenibilidad.

Como apoyo al desarrollo sostenible, la comunidad y el municipio no solo se limitan a reciclar, sino que pretenden transformar (por medio de inyección de plásticos) materiales para convertirlos en materia prima, con el fin de confeccionar artículos tales como tapas de alcantarillado o señales de tránsito, lo que deja ver su visión de largo plazo e innovación.

Con respecto a los residuos de envases multicapa, como los tetra brik, que por su composición química son sumamente difíciles de reciclar o reutilizar, se están realizando pruebas para fabricar láminas para la construcción denominadas Tectan, que pueden ser un producto sustituto verde de los materiales que existen actualmente en el mercado.

Es importante señalar que las láminas de Tectan son $100 \%$ reciclables, lo cual es un punto a favor, ya que la generación de residuos disminuye, se podrían generar fuentes de empleo al tratar el material y eso generaría un aporte económico para la comunidad. De igual manera, cuando se construya la planta procesadora para las láminas de Tectan, el municipio ha pensado en la reutilización del agua resultante del proceso, recuperándola por medio de floculación y/o coagulación de acuerdo con las características propias del líquido, lo que incidirá en la reducción de la huella hídrica. También se pretende que la planta de proceso cuente con los recipientes adecuados para que los colaboradores hagan una adecuada separación de los residuos que se generan propios de su actividad diaria. Las generaciones de $\mathrm{CO}_{2}$ de la planta provenientes de la electricidad y gasolina de los camiones, entre otros, se compensarán con la siembra de árboles en el terreno de la municipalidad y/o áreas cercanas.

En el caso de la Municipalidad de Jiménez, existe un reglamento vigente el cual indica que los residuos se recolectarán solamente sí se encuentran separados en bolsas de diferentes colores: verde para residuos orgánicos, transparente para inorgánicos reciclables y negra para inorgánicos no reciclables. Lo anterior se ha logrado con el desarrollo de los talleres realizados con los actores principales del cantón. Esto significa una separación discriminada en la fuente, ya que los residuos de la bolsa negra se llevan al relleno sanitario, los inorgánicos reciclables se aprovechan en el centro de acopio del cantón, en el cual laboran mujeres jefas de hogar o personas con algún grado de discapacidad, con el fin de brindar igualdad de oportunidades a toda la población.

Respecto a los residuos orgánicos, estos son transformados en abono orgánico, el cual se utiliza en las fincas de la localidad.

Todo lo anterior denota que ambas comunidades, en forma conjunta con los gobiernos locales, brindan un gran aporte al componente socioambiental, con la incorporación de grupos desfavorecidos socialmente, el aumento en los índices económicos de un sector de la población y un gran aporte al componente ambiental, debido a la disminución de residuos sólidos que se desvían de la corriente tradicional evitando así que lleguen al relleno sanitario, derivando en un impacto económico, social, ambiental y hasta cultural positivo para la comunidad.

Elizalde (2009) denomina este aprovechamiento de materias la estrategia de desmaterialización. A través de ella se pueden disminuir las salidas de residuos y sustancias tóxicas al medio ambiente, lo que podría permitir una reducción en la intensidad del uso de los materiales y energías, además de impulsar procesos de educación ambiental con estrategias como el reciclaje, que traerán beneficios para toda la sociedad. 
En este sentido, la Municipalidad de Jiménez, con la colaboración de sus habitantes, ha logrado recuperar cerca de 22 toneladas por mes de residuos biodegradables que se han transformado en abono.

Respecto a los residuos inorgánicos, como se observa en el Cuadro I, se ha logrado recuperar plástico, envases PET, latas de aluminio, chatarra, vidrio, tetra brik y cartón, lo cual representa un total aproximado de seis toneladas mensuales (datos suministrados por la Municipalidad de Jiménez, 2009).

Por otra parte, como se observa en el cuadro 2, la Municipalidad de Oreamuno, al igual que la de Jiménez, está acopiando papel, cartón, plástico, aluminio, tetra brik, los cuales se distribuyen a diferentes empresas. Esto ha significado que en el término de tres meses se ha creado un beneficio económico para la comunidad, además de evitarse que lleguen 29305 kilos al relleno sanitario, cantidad que se incrementará conforme avance el proyecto.

Lo anterior son ejemplos de trabajo participativo comunitario, que se realiza aplicando variables de conocimientos, habilidades, actitudes y barreras para coadyuvar a un plan de gestión de residuos sólidos.

Se evidencia así cómo la complejidad del ámbito de la gestión de residuos hace necesario el trabajo conjunto de la municipalidad con una amplia gama de actores, que se deben incorporar a los planes de manejo (CYMA, 20I0).

Cuadro I. Cantidades de algunos materiales recuperados en el Centro de Acopio de Jiménez, de abril a junio de 2009.

\begin{tabular}{|c|c|c|}
\hline Material & $\begin{array}{c}\text { Cantidad } \\
\text { recuperada }(\mathrm{kg})\end{array}$ & $\begin{array}{c}\text { Dinero recolectado } \\
\text { (colones) }\end{array}$ \\
\hline Papel y periódico & 757 & 21953 \\
\hline Cartón & 4450 & 120150 \\
\hline Vidrio & 4680 & 131040 \\
\hline Chatarra & 5506 & 214525 \\
\hline Plástico & 2536 & 106155 \\
\hline Aluminio & 109 & 50200 \\
\hline tetra brik & 534 & 18690 \\
\hline Total & 18572 & 662713 \\
\hline
\end{tabular}

Fuente: Datos suministrados por la Municipalidad de Jiménez.
Para obtener un buen conocimiento del papel que cada uno de los actores tiene en la gestión de residuos, debe analizarse la función y alcance de cada uno de ellos para aprovechar sus experiencias, conocimientos y grado de autoridad en la búsqueda de un mejor ambiente (Wehenpohl y Hernández, 2002).

Apoyando lo anterior, Gallopín (2003) menciona que para alcanzar la sostenibilidad se debe lograr la participación integral de la sociedad y el ecosistema.

La importancia de involucrar a la mayoría de los actores en el manejo integral de los residuos sólidos también radica en que el problema ambiental no tiene barreras, independientemente de la cultura, religión, país de procedencia o color de piel. Esto es lo que menciona Beck (2004), al decir que "Tanto si pensamos en los miedos al agujero en la capa de ozono, la polución o la comida, la naturaleza está indisociablemente contaminada por la actividad humana. Esto es, el peligro común tiene un efecto igualador que reduce las barreras cuidadosamente levantadas entre clases, naciones, humanos y el resto de la naturaleza". Lo anterior es lo que se conoce como "la teoría de sociedad del riesgo", la cual, como indica Soto (2009), constituye "una teoría sociológica que, a diferencia de las anteriores, trata de explicar lo que sucede actualmente en el mundo a partir de lo ecológico o ambiental. Pero lo ecológico no se limita solamente a plantas y animales, reino vegetal y reino animal, sino que incluye hombres y mujeres".

Cuadro 2. Cantidades de algunos materiales recuperados en el Centro de Acopio de Oreamuno, de julio a octubre de 2010.

\begin{tabular}{|c|c|c|}
\hline Material & $\begin{array}{c}\text { Cantidad } \\
\text { recuperada }(\mathrm{kg})\end{array}$ & $\begin{array}{c}\text { Dinero recolectado } \\
\text { (colones) }\end{array}$ \\
\hline Papel y periódico & 11557 & 492530 \\
\hline Cartón & 8005 & 180650 \\
\hline Vidrio & 7812 & 194390 \\
\hline Latón & 645 & 12900 \\
\hline Hierro & 98 & 7350 \\
\hline Plástico & 464 & 25000 \\
\hline Aluminio & 245,5 & 105400 \\
\hline Tetra brik & 506 & 19105 \\
\hline Total & 29305,5 & 1037325 \\
\hline
\end{tabular}

Fuente: Quirós, 2011. 
Sin embargo, para concientizarse sobre los riesgos potenciales a los que se podría enfrentar una comunidad, es necesario primero reconocer que el problema del mal manejo de los residuos sólidos es global, por lo cual es responsabilidad de la sociedad llevar a cabo acciones de mitigación, como las que están realizando las comunidades de Oreamuno y Jiménez.

Conociendo que un pilar importante del desarrollo sostenible se refiere al componente económico y específicamente a la rentabilidad de los proyectos, en el caso de estos dos municipios se podría pensar que la recuperación económica aún es poca. No obstante, si se piensa en la generación de empleo que ha permitido a las personas sentirse parte del proceso económico, la contribución que se está brindando desde la perspectiva de educación ambiental, al sensibilizar y empoderar a las personas por medio del trabajo comunitario en pro de la sociedad y a esto se le suman los costos evitados en el tratamiento alternativo de residuos al brindar una opción para que los residuos sólidos valorizables no lleguen a un vertedero o botadero a cielo abierto, se podría estar al frente de posibles beneficios directos (externalidades positivas), dentro de los cuales se podría mencionar que es una alternativa tecnológica sostenible para el manejo de los residuos sólidos en ambos cantones, que implica reducción de emisiones de $\mathrm{CO}_{2}$, lixiviados, costos operativos y menor consumo de combustible al no tener que transportar los desechos hasta un vertedero o relleno sanitario.

Todo lo expuesto anteriormente refleja el grado de compromiso de dos municipalidades y sus habitantes en la búsqueda del desarrollo y la sostenibilidad. Por medio de acciones estandarizadas y basadas en el Modelo de Acción Ambienta Positiva, demuestran que es posible superar las barreras usando el conocimiento, lo cual saca a flote las habilidades y destrezas de las personas, lo que emparejado con un cambio de actitud permite el empoderamiento y la pertenencia. Ello se refleja en que las comunidades concientizadas y empoderadas son capaces de llevar a cabo, en conjunto con los actores institucionales, la gestión integral de los residuos sólidos.

\section{Conclusiones}

Los municipios Jiménez y de Oreamuno han trabajado en los conocimientos, habilidades, destrezas, actitudes, sensibilidad y el empoderamiento por medio de un modelo de Acción Ambiental Positiva, logrando el tratamiento y disposición adecuada de los residuos sólidos.

El cantón de Oreamuno cuenta con un Plan de Manejo Integral de Residuos Sólidos que ha reducido al mínimo los desechos, que de otra manera irían al relleno sanitario. Asimismo, se gestiona la recuperación de gases y la remoción de los metales en los lixiviados utilizando métodos químicos y biológicos, además de la recuperación, comercialización y transformación de los residuos reciclables y reutilizables, generando oportunidades de empleo.

El cantón de Jiménez está separando y seleccionando los residuos orgánicos e inorgánicos; los inorgánicos reciclables son aprovechados en el centro de acopio propiciando la participación inclusiva de personas discapacitadas y mujeres jefas de hogar. Los residuos orgánicos son transformados en abono orgánico que se utiliza en las fincas de la localidad.

Por último, se han creado beneficios sociales como la generación de empleo a sectores vulnerables y se ha iniciado un proceso de sensibilización de la población por medio de las acciones en educación ambiental y la incorporación de los diferentes actores involucrados, ya que ellos son los gestores de una ciudad desarrollada y sostenible.

De esta manera, los municipios de Oreamuno y Jiménez dan un ejemplo ante el mundo y ante las personas como individuos de una sola sociedad global, siendo conscientes de que los riesgos ambientales afectan a todas las personas por igual. Es por ello que el impacto social y ambiental de las acciones que han emprendido estas dos comunidades costarricenses las convierten en un ejemplo a seguir.

\section{Bibliografía}

Beck, U. (2004). Poder y contra poder en la era global. La nueva economía política mundial. España: Paidós (Capítulo VIII: Pequeño discurso fúnebre en la cuna de la era cosmopolita). 
Caduto, M. (1992). Guía para la enseñanza de valores ambientales. Programa Internacional de Educación Ambiental. Madrid: UNESCO-PNUMA.

CYMA-Programa Competitividad y Medio Ambiente. (2007). Plan de Residuos Sólidos: Diagnóstico y áreas prioritarias. San José, 220 p.

CYMA-Programa Competitividad y Medio Ambiente. (2010). Manual para el diseño y formulación de Reglamentos Municipales de Gestión Integral de Residuos Sólidos. San José, 45 p.

Elizalde, A (2009). Qué desarrollo puede llamarse sostenible en el siglo XXI. Revista de Educación. Número extraordinario. Santiago de Chile.

Gallopín, G. (2003). Sostenibilidad y desarrollo sostenible: un enfoque sistémico. Chile: CEPAL.

García, A. (2006). Diagnósticos y potenciación de la educación ambiental en jóvenes universitarios. Revista electrónica de pedagogía 3(6), 3-32.

Gaviria, L. \& Soto, S. (2008). Situación de la gestión de residuos sólidos en las municipalidades de Costa Rica; gestión ambiental municipal. Tecnología en Marcha, Vol. II.

López, E., López, C. \& Rodríguez, A. (2005). Avances y perspectivas de un modelo de educación ambiental en la zona indígena chontal de Tabasco. México. Obtenido desde: http://anea.org.mx/eventos/comie2007/ Extenso/PonenciaLopezE_RodriguezA_RicaldeC.pdf

Mata, A., Zúñiga, C., Brenes, O., Carrillo, M., Charpentier, C., Hernández, L. \& Zúñiga, M. (2002). Dimensión ambiental. Estrategias innovadoras para la formación docente. CEEC. I ed. Vol. 27. Cartago, CR: Impresora Obando S.A.

Mata, A., Zúñiga, C., Brenes, O., Carrillo, M., Charpentier, C., Hernández, L. \& Zúñiga, M. (2009).
Dimensión Ambiental. Estrategias Innovadoras para la Formación Docente. San José: CECC.

Novo, M. (2009). La educación ambiental, una genuina educación para el desarrollo sostenible. Revista de Educación. ISSN 003 I-8082. Nºxtra I (pp. 195-2 I7. Recuperado el 30 de septiembre 201 I desde http:// dialnet.unirioja.es/servlet/articulo?codigo $=3019430$

Programa Estado de la Nación (2006). Duodécimo Informe Estado de la Nación en Desarrollo Humano Sostenible. San José: Programa Estado de la Nación.

Quirós, N. (20II). Estudio de prefactibilidad técnica para la fabricación de materiales aprovechables a partir de residuos de tetra pak del cantón de Oreamuno. Tesis. En: www.uci.ac.cr

Redondo, M. (2009). Plan de Manejo de Desechos Sólidos Municipales. Municipalidad de Oreamuno.

Soto, S. (2006). Situación actual de la gestión de los residuos sólidos en Costa Rica. Ponencia preparada para el Duodécimo Informe Estado de La Nación. San José: Programa Estado de la Nación.

Soto, W. (2009). La teoría de la sociedad del riesgo global: ies capaz de dar cuenta del acontecer mundial actual? Boletín del Observatorio de la Política Exterior, Año 3, Número I I. Costa Rica: Universidad Nacional y Universidad de Costa Rica.

Soto, S. (2010). Generación y Manejo de Residuos Sólidos. (en línea). Décimo sexto informe Estado de la Nación en Desarrollo Humano Sostenible. Consultado 21 abril 20ll. Disponible en: http:// www.estadonacion.or.cr/images/stories/informes/0 I 6/Armonia/silvia_soto.pdf

Wehenpohl, G. \& Hernández, C. (2002). Guía en la Elaboración de Planes Maestros para la Gestión Integral de los Residuos Sólidos Municipales. México: Secretaría de Ecología del Gobierno del Estado de México. 